$$
\text { Pontifícia Universidade C Católica }
$$

Daniel Brantes Ferreira

\title{
Realismo Jurídico Norte-Americano: Uma contribuição para o ensino jurídico
}

\author{
Tese de Doutorado
}

Tese apresentada como requisito parcial para obtenção do título de Doutor pelo Programa de PósGraduação em Direito da PUC-Rio.

Orientadora: Profa. Nadia de Araujo

Rio de Janeiro

Junho de 2011 


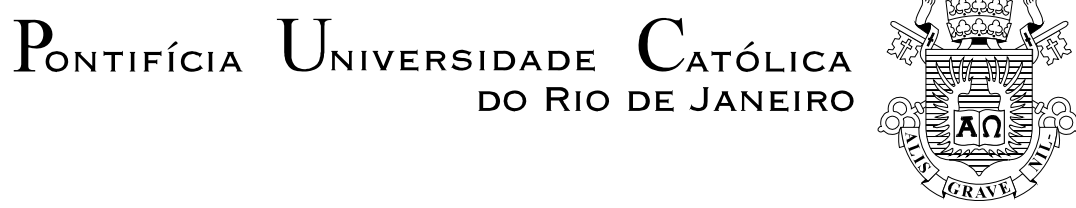

Daniel Brantes Ferreira

\section{Realismo Jurídico Norte-Americano: Uma contribuição para o ensino jurídico}

Tese apresentada como requisito parcial para obtenção do título de Doutor pelo Programa de Pós-graduação em Direito do Departamento de Direito da PUC-Rio. Aprovada pela Comissão Examinadora abaixo assinada.

Prof $^{\text {. Nadia de Araujo }}$
Orientadora
Departamento de Direito - PUC-Rio

Prof. Noel Struchiner

Co-orientador

Departamento de Direito - PUC-Rio

Profa. Daniela Trejos Vargas Departamento de Direito - PUC-Rio

Profa. Gisele Guimarães Cittadino

Departamento de Direito - PUC-Rio

Prof. Carlos Eduardo de Abreu Boucault

UNESP

Profa. Maria Guadalupe Piragibe da Fonseca

IBMEC - RJ

Profa. Mônica Herz

Vice-Decana de Pós-Graduação do Centro de

Ciências Sociais - PUC-Rio

Rio de Janeiro, 13 de junho de 2011. 
Todos os direitos reservados. É proibida a reprodução total ou parcial do trabalho sem autorização da universidade, do autor e do orientador.

\section{Daniel Brantes Ferreira}

Bacharel em Direito pela PUC-Rio (2000-2004). Mestre em Direito Constitucional e Teoria do Estado pela PUC-Rio (2005-2007). Possui como principais áreas de estudo a teoria geral do Direito, o ensino jurídico, teoria da constituição, hermenêutica jurídica e filosofia do Direito. Além disso, durante o segundo semestre de 2009 (Agosto a Dezembro) foi pesquisador visitante na Law School da State University of New York at Buffalo na qualidade de bolsista sandwich da CAPES (PDEE) onde realizou pesquisa para sua tese de doutorado sobre realismo jurídico norte-americano e ensino jurídico norte-americano

Ficha catalográfica

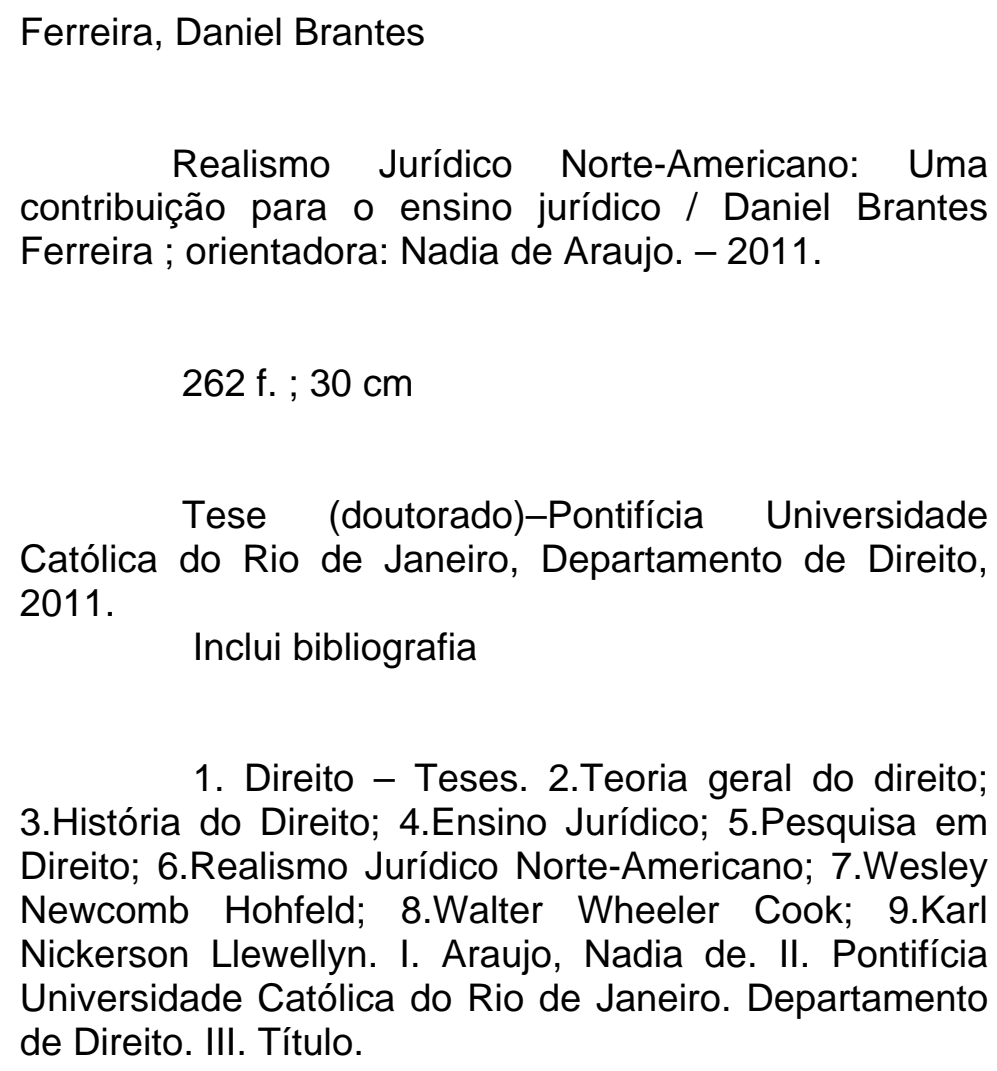

1. Direito - Teses. 2.Teoria geral do direito; 3.História do Direito; 4.Ensino Jurídico; 5.Pesquisa em Direito; 6.Realismo Jurídico Norte-Americano; 7.Wesley Newcomb Hohfeld; 8.Walter Wheeler Cook; 9.Karl Nickerson Llewellyn. I. Araujo, Nadia de. II. Pontifícia Universidade Católica do Rio de Janeiro. Departamento de Direito. III. Título. 
A minha mãe Suely, sempre. 


\section{Agradecimentos}

Ao Professor John Henry Schlegel da University at Buffalo Law School sem o qual a presente tese tornar-se-ia inviável. Gentilmente o Professor dividiu todo seu conhecimento e bibliografia com um estudante brasileiro que nem conhecia. A ele minha admiração e meu eterno agradecimento.

Aos meus orientadores brasileiros pelo apoio de sempre: Professora Nadia de Araújo e Professor Noel Struchiner.

Ao meu irmão Jorge Brantes Ferreira e ao meu pai Jorge Ferreira da Silva, dois eméritos professores de administração de empresas que sempre me ajudaram com valiosos conselhos acadêmicos e com seu amor.

A minha noiva, Deborah Nunes pelo apoio e amor incondicionais em todos os momentos da elaboração deste trabalho.

Ao Anderson e a Carmen, melhores secretários de pós-graduação que um programa poderia ter.

Ao programa de pós-graduação em Direito da PUC-Rio que possibilitou o meu desenvolvimento acadêmico.

A CAPES pelo apoio financeiro tanto em solo nacional quanto estrangeiro. 


\section{Resumo}

Ferreira, Daniel Brantes; Araujo, Nadia de. Realismo Jurídico NorteAmericano: Uma contribuição para o ensino jurídico. Rio de Janeiro, 2011. 262p. Tese de Doutorado - Departamento de Direito, Pontifícia Universidade Católica do Rio de Janeiro.

A tese tem como hipótese a importância do realismo jurídico norteamericano para o desenvolvimento do ensino jurídico e das escolas de direito norte-americanas. Para isto três autores serão abordados: Wesley N. Hohfeld, Walter W. Cook e Karl N. Llewellyn. O primeiro capítulo trará uma introdução sobre as questões metodológicas do trabalho. O segundo capítulo fará uma digressão histórica no direito norte-americano para podermos compreender o contexto de surgimento do realismo jurídico. O terceiro capítulo abordará Wesley N. Hohfeld, sua importância para o surgimento do movimento realista e também sua contribuição para a evolução do ensino jurídico norte-americano. O quarto capítulo tratará de Walter W. Cook, sua teoria e sua experiência fracassada no Instituto para o Estudo de Direito de Johns Hopkins. O quinto capítulo tratará especificamente do surgimento do realismo jurídico através dos escritos de Karl N. Llewellyn e também de sua teoria das funções do direito. A crítica de Llewellyn ao ensino jurídico da época será ponto central do capítulo. O sexto capítulo demonstrará o porquê de o movimento realista ter sucumbido tão rápido, bem como abordará o seu legado. Finalmente a conclusão demonstrará as limitações do trabalho e suas principais contribuições.

\section{Palavras-chave}

Teoria geral do direito; História do Direito; Ensino Jurídico; Pesquisa em Direito; Realismo Jurídico Norte-Americano; Wesley Newcomb Hohfeld; Walter Wheeler Cook; Karl Nickerson Llewellyn. 


\section{Abstract}

Ferreira, Daniel Brantes; Araujo, Nadia (Advisor). American Legal Realism: A contribution to legal education. Rio de Janeiro, 2011. 262p. Doctoral Thesis - Departamento de Direito, Pontifícia Universidade Católica do Rio de Janeiro

The thesis has as its main hypothesis the importance of American legal realism to the development of American Law Schools and legal education. In order to accomplish that, three authors will be faced: Wesley N. Hohfeld, Walter W. Cook e Karl N. Llewellyn. The first chapter will bring an introduction about the methodological issues of the present work. The second chapter will make a historical digression on the American law so that the reader can understand in which context American legal realism appeared. The third chapter will be about Wesley N. Hohfeld and his importance to the appearance of American legal realism and also his contribution to legal education. The fourth chapter will treat Walter W. Cook's theory and his failure in the John's Hopkins Institute. The fifth chapter will treat specifically about the appearance of the legal realism movement through Karl N. Llewellyn articles and his law-jobs theory. Also, his criticism about legal education at the time will be the core of this chapter. The sixth chapter will demonstrate why American legal realism was not successful in its attempt and why it didn't last long. It also will demonstrate the important legacy left by the movement. Finally, the conclusion will demonstrate the thesis's limitations and its main contributions.

\section{Keywords}

Legal Theory; Legal History; Legal Education; Legal Research; American Legal Realism; Wesley Newcomb Hohfeld; Walter Wheeler Cook; Karl Nickerson Llewellyn. 


\section{SUMÁRIO}

1. Introdução 11

1.1. A hipótese 11

1.2. Metodologia aplicada 13

1.3. Estrutura do trabalho 14

2. Ensino e prática jurídica nos EUA: Uma evolução histórica 16

2.1. A era do descobrimento (1787 - 1860 - Guerra Civil americana 16

2.2. A era da fé (1860-1914 - Da Guerra Civil até a Primeira Grande 23

Guerra)

2.3. A era da ansiedade (1914 até os dias de hoje - Primeira Grande 31

Guerra até os dias atuais)

3. Wesley Newcomb Hohfeld: Principal influência do movimento 36 realista

3.1. Vida e obra

36

3.2. Escola de Direito ideal - Hohfeld e o Ensino Jurídico 43

3.2.1. O estudo sistemático e sobre o desenvolvimento dos sistemas 45

jurídicos (The systematic and Developmental Study of Legal Systems)

3.2.1.1. História ou genética do Direito (Historical, or genetical, 47 jurisprudence)

3.2.1.2. Direito comparado ou eclético (Comparative, or eclectic, 48 jurisprudence)

3.2.1.3. Direito formal ou analítico (Formal, or analytical, 50

jurisprudence)

3.2.1.4. Direito crítico ou teleológico (Critical, or teleological, 51 jurisprudence)

3.2.1.5. Direito legislativo ou construtivo (Legislative, or constructive 52 jurisprudence)

3.2.1.6. Direito dinâmico ou funcional (Dynamic, or functional, 53 jurisprudence)

3.2 .2

O estudo profissional e detalhado do Sistema jurídico Anglo-

11

3

4

6

16

Americano (The Professional and Detailed Study of the AngloAmerican System)

3.2.3. O estudo cívico e cultural das instituições jurídicas (The Civic and Cultural Study of Legal Instituitions)

3.2.4. A questão financeira

3.3. Wesley N. Hohfeld como principal influência de Walter W. Cook

3.4. Hohfeld - inspiração realista a contragosto

3.4.1. Joseph W. Bingham e seu artigo (What is the Law? - 1912) 
4. Walter Wheeler Cook - Grande influência do realismo jurídico 71

4.1. Vida e obra

4.2. Walter W. Cook e o Ensino Jurídico - Sua experiência em Johns 73

Hopkins

4.3. A Teoria do Direito de Cook - o método científico aplicado ao 79

Direito

4.3.1. Principais influências teóricas de Cook 79

4.3.2. O método científico aplicado ao Direito 83

4.3.3.Cook - Empirismo científico $\quad 89$

4.4.Walter W. Cook como influência de Karl N. Llewellyn 92

5.Karl N. Llewellyn e o realismo jurídico 95

5.1. Vida e obra 95

5.2. Karl N. Llewellyn e o movimento realista 100

5.2.1. Roscoe Pound e seu artigo The Call for a Realist 104

Jurisprudence - A crítica ao Realismo Jurídico de Llewellyn

$\begin{array}{ll}\text { 5.3. Karl Llewellyn e o ensino jurídico } & 119\end{array}$

5.3.1. The Bramble Bush - O que oferece a Escola de Direito? 121

5.3.2. O que estaria errado com o ensino jurídico? 124

5.3.3. Relatório sobre o ensino jurídico (1945) 129

5.4. A Teoria do Direito de Llewellyn: as funções do direito (Law-jobs) 133

6. A derrocada do realismo jurídico e o seu legado teórico 141

6.1. A derrocada 142

6.2. O legado 145

7. Conclusão 157

7.1. Principais contribuições da tese 157

7.2. Limitações da tese 158

7.3. Propostas para pesquisas futuras 159

8. Referências bibliográficas 160

8.1. Sítios consultados 169

9. ANEXOS: (Cartas de Wesley N. Hohfeld para Roscoe Pound - 170 $1910 / 1918$ 


\section{Epígrafes:}

"It is impossible to write about intellectual history, but it is possible to write about the history of intellectuals”. (John Henry Schlegel) ${ }^{1}$

"My brother Gray," runs one garbled version of a story, "has taught you what the law Used to be; my brother Ames has taught you what the law Ought to be; I intend, with your indulgence, to give some attention to what the law Is." (Karl N. Llewellyn $)^{2}$

"When we become too sure of our premises, we necessarily fail in what we are supposed to be doing" (Grant Gilmore) ${ }^{3}$.

\footnotetext{
${ }^{1}$ SCHLEGEL, John H.. Assim que chegamos a Buffalo, NY, no dia 07.08.2009, e começamos a conversar sobre realismo jurídico norte-americano com o professor Schlegel essa foi uma das primeiras frases que ele disse. Realmente é impossível escrever sobre realismo jurídico norteamericano de forma abstrata. O trabalho só é possível intercalando as vidas, trajetórias, e pensamentos daqueles que viveram aquele momento histórico. Em tradução livre: "É impossível escrever sobre história da intelectualidade. Porém, é possível escrever sobre a história dos intelectuais".

${ }^{2}$ LLEWELLYN, Karl N.. On What is Wrong with So-Called Legal Education in Columbia Law Review, vol. 35, 1935, p. 672.

${ }^{3}$ GILMORE, Grant. The Ages of American Law. Connecticut: Yale University Press, 1977, p. 110.
} 